\title{
LACUNARITY ANALYSIS OF FOSSIL DISTRIBUTION PATTERNS AND IMPLICATIONS FOR THE ESTIMATION OF BIOSTRATIGRAPHIC RANGES
}

PLOTNICK, Roy E., Department of Geological Sciences, University of Illinois at Chicago, 845 W. Taylor St., Chicago, IL 60607, U.S.A.

Lacunarity analysis is a multi-scaled method for describing patterns of spatial dispersion. It measures the extent to which the distribution of some property of interest, such as the occurrence of fossils, deviates from regularity over a range of scales. It can be used with both binary and quantitative data in 1-, 2-, and 3-dimensions. Lacunarity analysis is broadly applicable to many data sets of paleontological interest. It can be used to describe the spatial distribution of fossiliferous horizons and determine whether the distribution can be modeled as random, regular, fractal, or clumped. Similarly, it can be used to determine if fossil abundance patterns are random or follow some other distribution, such as a multifractal one. Lacunarity analysis can also be used to reveal scale-related changes of pattern. For example, it can be used to determine if fossil occurrences are clumped at one scale and random on another. Lacunarity analysis is computationally simple and provides readily interpretable graphic results.

Mathematical models for confidence intervals on biostratigraphic ranges have heavily relied on the assumption of randomness of distribution within the range (although recent work by Marshall (1994) points to a relaxation of this assumption). These techniques evaluate confidence intervals based on the total number of fossil horizons and do not consider the distribution of horizons within the range. In addition, tests for violation of the assumption of randomness do not distinguish among different patterns of deviation from randomness. Lacunarity analysis, by allowing the detailed characterization of patterns of fossil occurrence, can suggest alternative models, such as fractal ones, for the estimation of confidence intervals.

Lacunarity analysis is illustrated for datasets drawn from DSDP/ODP reports and from the Ordovician of Indiana (Cummings and Galloway, 1913). Most analyses reveal that stratigraphic distributions differ from randomness by being clumped. This is not unexpected, given the strong facies and taphonomic controls over fossil occurrences (for example, Holland, 1995). 